\title{
Factors Predictive of Failure to Complete Planned Intraoperative Breast Radiation Using the Intrabeam ${ }^{\circledR}$ System
}

\author{
Running Title: Failure to complete planned breast IORT \\ Lesly A Dossett MD MPH${ }^{1}$, Andrea M Abbott MD ${ }^{1}$, Weihong Sun MD ${ }^{1}$, Loretta Loftus MD ${ }^{1}$, \\ $\mathrm{M}$ Catherine Lee $\mathrm{MD}^{1}$, Roberto Diaz MD $\mathrm{PhD}^{1,2}$, Christine Laronga $\mathrm{MD}^{1}$ \\ ${ }^{1}$ Comprehensive Breast Program \\ ${ }^{2}$ Department of Radiation Oncology \\ H. Lee Moffitt Cancer Center \& Research Institute, Tampa, Florida USA
}

This data was presented in poster format at the American College of Surgeons Clinical Congress October 2015 (Chicago, IL).

Corresponding Author:

Lesly A Dossett MD MPH

Comprehensive Breast Program

H. Lee Moffitt Cancer Center 10920 N McKinley Drive, $5^{\text {th }}$ Floor

Tampa, Florida 33612

813-745-8238 (phone)

813-449-8210

lesly.dossett@moffitt.org

Synopsis: Insufficient skin-to-applicator spacing is the most common reason for failure to complete planned IORT. In this series, higher volume surgeons completed a greater proportion of IORT cases, suggesting a learning curve to patient selection or intraoperative technique.

\section{Conflict of Interest Notification}

None of the authors have any conflicts of interest related to this manuscript.

\begin{abstract}
Purpose. Intraoperative radiation therapy (IORT) is a form of breast irradiation that is delivered in a single session at the time of partial mastectomy. In up to $10 \%$ of patients, planned IORT is not completed; this leads to wasted resources and decreased patient satisfaction. Our objective was to evaluate factors associated with failure to complete planned IORT.
\end{abstract}

Methods and Materials. An IRB-approved review of planned IORT cases from 2011 to 2015 was conducted. Eligibility criteria included: age $\geq 60$, invasive ductal or mammary carcinoma, 
tumor $<3.0 \mathrm{~cm}$, ER positive and clinically node negative. Discontinuation of planned IORT was at the discretion of the breast surgical and radiation oncologists.

Results. 21 (15\%) of 145 planned IORT cases were not completed. Reasons for failure to complete IORT included inadequate applicator to skin distance $(n=15,71 \%)$, altered wire localization findings the day of surgery $(n=4,19 \%)$, equipment failure $(n=1,5 \%)$ and hemodynamic instability $(\mathrm{n}=1,5 \%)$. Significant surgeon variability was associated with failure to complete planned IORT $(\mathrm{p}<0.001)$.

Conclusions. Insufficient skin-to-applicator spacing is the most common reason for failure to complete IORT. In this series, higher volume surgeons completed a greater proportion of IORT cases, suggesting a learning curve to patient selection or intraoperative technique.

\section{Key words: breast cancer, IORT, APBI Introduction}

Breast conserving surgery (BCS) followed by adjuvant whole breast external beam radiotherapy (EBRT) is a well-established option for patients with early-stage breast cancer (1-3). Adjuvant radiation totaling 45 to $50 \mathrm{~Gy}$ is typically delivered to the whole breast and administered in daily fractions for 5 consecutive weeks. This dose may be supplemented by an additional boost of 10 to 16 Gy delivered to the tumor bed to further improve local control. For some patients, the financial and physical burden of daily travel may be taxing or even prohibitive, leading these patients to decline adjuvant radiation after partial mastectomy or choose mastectomy in order to avoid the need for radiation. Accelerated partial breast irradiation (APBI), including intraoperative radiation therapy (IORT), is increasingly accepted as an alternative to whole breast EBRT in selected patients with favorable tumor features. In these settings, IORT is associated with adequate local control and minimal morbidity $(4,5)$.

In patients with early stage breast cancer, IORT may increase the utilization of radiotherapy by administering a single dose of radiation at the same time as BCS. IORT is 
planned in advance and requires coordination between the surgeon and radiation oncologist. In up to $7-11 \%$ of patients, planned IORT is not completed due to patient, equipment, or system factors $(5,6)$. Failure to complete planned IORT may lead to wasted resources (scheduled OR time and physician availability) and decreased patient satisfaction or compliance with recommended radiation. Improved patient and tumor selection or surgeon experience may help eliminate the failure to complete IORT. What is unknown is which factors-patient, clinical, system or provider-level—are associated with a failure to complete planned IORT, and if those factors can be identified or mitigated preoperatively. We sought to explore associations among these factors and failure to complete planned IORT at our institution through a retrospective review of all planned breast IORT cases.

\section{Methods and Materials}

We conducted an IRB-approved retrospective review of 145 consecutive patients with early stage breast cancer who elected breast conservation therapy with BCS and IORT from January 2011 to January 2015 at Moffitt Cancer Center (Tampa, FL). Eligible patients included those aged 60 or older with the following clinical and pathologic criteria: (1) a pathologic diagnosis of invasive ductal or mammary carcinoma (mixed ductal and lobular components) less than or equal to $3.0 \mathrm{~cm},(2)$ a clinically node negative axilla, (3) estrogen receptor (ER) positive and (4) the technical feasibility to accommodate a radiation applicator with a skin to applicator surface distance of at least $7 \mathrm{~mm}$. Patients who had received neoadjuvant chemotherapy were excluded. Eligibility for IORT was ultimately at the discretion of the breast surgeon and radiation oncologist after discussion at a breast multidisciplinary tumor conference.

The Intrabeam ${ }^{\mathrm{TM}}$ system (Figure 1) delivers low-energy photons (maximum $50 \mathrm{kV}$ ) at the tip of a $3.2 \mathrm{~mm}$ diameter tube. Spherical applicators of various sizes $(2-6 \mathrm{~cm})$ cover the tube and are placed in the tumor bed. The surface of the applicator receives $20 \mathrm{~Gy}$ and the absorbed dose rapidly attenuates to 5-7 Gy at $1 \mathrm{~cm}$ depth. The surgical technique for IORT with the Intrabeam ${ }^{\mathrm{TM}}$ 
system has been previously described(4). All patients undergo wire localization by either preoperative ultrasound or stereotactic guidance and receive a preoperative radiotracer injection for sentinel lymph node biopsy (SLNB). At the SLNB is completed and specimens are sent for intraoperative histologic review. The wire localized partial mastectomy is then completed, and a specimen radiograph is obtained. Gross pathologic evaluation of margins is also performed at the time of the operation. Any margin grossly less than $5 \mathrm{~mm}$ is re-excised prior to placement of the IORT applicator. Following confirmation of grossly negative margins and a negative SLNB on touch prep, the Intrabeam ${ }^{\mathrm{TM}}$ delivery system is placed into the partial mastectomy cavity. Intraoperative ultrasound is performed to ensure tissue conformance with the applicator and a skin-to-device distance of at least $7 \mathrm{~mm}$. Skin to applicator distance is measured sonographically in the superior, inferior, lateral and medical locations. Skin edges are retracted at least $1 \mathrm{~cm}$ from the applicator surface-shaft using the Lone Star Retractor System (Cooper-Surgical Inc, Stafford, TX). IORT is then delivered to achieve 20 Gy of targeted radiation to the partial mastectomy cavity. The treatment ranges from 15 to 25 minutes, depending on radiation applicator size. After completion of IORT, the partial mastectomy cavity is marked with surgical clips and the incision is closed in layers.

Failure to completed planned IORT was at the discretion of the attending breast surgeon with the radiation oncologist and was made the day of surgery. Planned IORT was defined as a plan to deliver IORT on the day of surgery, and failure to completed IORT did not include cases that were rescheduled due to discussion at multidisciplinary tumor conferences or changes due to patient choice.

For patients completing IORT, if final pathology confirmed histologically that margins and SLNB were negative, no further radiation therapy was administered. For cases of positive margins on final pathology (defined as tumor on ink), surgical re-excision was performed. In cases where residual tumor was present in the re-excision specimens (despite negative true 
margins) patients were treated with whole breast irradiation. These cases were not considered as failures to complete IORT, as the IORT dose served as the boost dose of radiation.

Descriptive statistics were performed. Normally distributed continuous variables were reported as mean and standard deviation and compared using a student $t$-test. Non-normally distributed variables were summarized by median and interquartile range (IQR) and compared using the Wilcoxon rank sum test. Categorical variables are reported as proportions and compared with a chi-square test. A p-value of 0.05 (two-sided test) was declared as significant. All analyses were conducted using Stata 14.0 (StataCorp, College Station, TX).

\section{Results}

A total of 145 consecutive planned IORT cases were reviewed. Of these cases, 21 (15\%) were not completed as planned due to a decision made the day of surgery. The demographic, clinical and pathologic characteristics of cases resulting in failure to complete planned IORT compared to those where IORT was completed are summarized in Table 1 . Age $(\mathrm{p}=0.35)$ and tumor size $(\mathrm{p}=0.40)$ did not differ between groups. Failure to complete planned IORT was associated with an increased need to take additional intraoperative margins based on gross examination by pathology; additional margin excision occurred in $48 \%$ of cases where IORT was completed and $81 \%$ of cases for which planned IORT was not completed $(\mathrm{p}=0.005)$. The intraoperative decision to take additional margins was based on the findings of any margin less than $5 \mathrm{~mm}$ on the gross evaluation. Tumors that required additional intraoperative margins were slightly larger (median $10 \mathrm{~mm}$ versus $8 \mathrm{~mm}$ for no additional margins, $\mathrm{p}=0.02$ ). After adjusting for tumor size, the need for additional intraoperative margins was not associated with failed IORT (OR $0.63,95 \%$ CI $0.18-2.13, \mathrm{p}=0.46$ ). A majority of patients who did not complete planned IORT $(81 \%)$ went on to receive postoperative whole breast EBRT whereas only $10 \%$ of those completing planned IORT required postoperative radiation (due to findings on final pathology). 
Women receiving EBRT after IORT were treated for 1) persistently positive margins requiring re-excision, 2) pathologic node positive disease, and 3) a change in histologic diagnosis.

Reasons for failure to complete planned IORT are summarized in Table 2 and included inadequate skin-to-applicator distance after partial mastectomy $(\mathrm{n}=15,71 \%)$, altered wire localization findings on the day of the procedure $(n=4,19 \%)$, equipment failure $(n=1,5 \%)$, and hemodynamic instability $(n=1,5 \%)$. When stratifying by surgeon, there were considerable differences in rates of failed IORT with rates ranging from 3-50\% ( $\mathrm{p}<0.001$, Table 3). Surgeons with the highest volume of IORT cases had the lowest rates failure to completed planned IORT.

\section{Discussion}

Despite the overall excellent results obtained with breast conserving surgery (BCS) followed by whole breast EBRT, there is growing interest in APBI as a means to reduce the treatment and travel burden associated with five weeks of daily radiation treatments. This is especially true for elderly patients, who are more likely to have favorable tumor biology and for whom the travel burden may be particularly arduous. In patients with early stage tumors and favorable characteristics, IORT offers the opportunity to complete radiation therapy in a single setting at the same time as partial mastectomy, potentially increasing the overall utilization of adjuvant radiation among this cohort. Executing IORT requires preoperative coordination with the surgeon, radiation oncologist and radiation physicist. Failure to complete planned IORT can lead to wasted time and resources, and be costly for both the patient and the health system.

In our series, planned IORT was not completed in $17 \%$ of cases, which is comparable to the $11 \%$ reported in the TARGIT-A trial(5). The most common reason for failure to completed planned IORT was a skin to applicator distance of less than $0.7 \mathrm{~cm}$. In $81 \%$ of these cases, compared to only $48 \%$ of completed cases $(\mathrm{p}=0.005)$, the surgeon took additional intraoperative margins based on gross pathologic examination of margin less than $5 \mathrm{~mm}$. The need to take 
additional surgical margins potentially decreases the skin-to-applicator distance. One explanation for the need for additional intraoperative margins is a larger tumor size, which was noted in this series. While the difference in median tumor size was not significantly different between groups, tumor size was larger in cases requiring additional margins $(\mathrm{p}=0.02)$. Accurate localization or use of the localization wire in surgery may also play a role, as $50 \%$ of cases requiring additional intraoperative margins were for tumors less than $0.7 \mathrm{~cm}$ in size. Several techniques can be used to potentially increase the skin-to-applicator distance including purse-string suture placement, tissue rearrangement and bolus saline injection. These techniques may help achieve an adequate skin-toapplicator distance, but could also contribute to local ischemia which could increase skin toxicity rates.

Provider variability was also associated with failure to complete planned IORT. Several possible factors may account for this finding. Most likely is that volume of experience with IORT may improve either patient selection or lumpectomy technique needed to achieve an adequate skin-to-applicator distance. In particular, the two-dimensional nature of mammography and the operator-dependent differences in ultrasound play a significant role in patient selection, but are only estimates of the actual skin-to-applicator distances observed intraoperatively. Variability among radiation oncologists may also contribute to these findings, as the initial recommended skin spacing mandated $10 \mathrm{~mm}$ from skin to applicator, and gradually decreased to $7 \mathrm{~mm}$ over time. However, the $7 \mathrm{~mm}$ distance was not uniformly adopted by all faculty and likely contributed to discontinuation of planned therapy intraoperatively in some cases.

Another consideration is that a small percentage of patients may have unexpected findings on imaging at the time of wire localization, such as lesions that appear multi-focal or larger than appreciated on initial imaging. In our series this occurred in 19\% $(n=3)$ of the failed cases ( $2 \%$ of total IORT cases). In these scenarios, the initial imaging had been performed outside our institution. Careful preoperative imaging may help to eliminate this as a cause of failure to complete IORT. MRI may be warranted in selected cases where the tumor approximates the 
anterior (superficial) margin, but the low incidence would not provide justification for routine preoperative MRI if not otherwise indicated.

The strengths of the study include well-established selection criteria and a consistent, uniform multidisciplinary treatment plan developed by fellowship trained breast specialists at a high volume cancer center. Despite these strengths, there are several important limitations. First, while this is a relatively large series of patients undergoing IORT at a single institution, this retrospective dataset remains underpowered for many outcomes and the overall median follow up is short (24 months). Second, while the single center design allows for uniformity in selection criteria and application of IORT, it limits the generalizability of the data to other centers.

\section{Conclusion}

Insufficient skin-to-applicator spacing is the most common reason for failure to complete IORT. In this series, higher volume surgeons completed a greater proportion of IORT cases, suggesting a learning curve to patient selection or intraoperative technique.

\section{References}

1. Clarke M, Collins R, Darby S, Davies C, Elphinstone P, Evans V, Godwin J, Gray R, Hicks C, James S, MacKinnon E, McGale P, McHugh T, Peto R, Taylor C, Wang Y, Early Breast Cancer Trialists' Collaborative G. Effects of radiotherapy and of differences in the extent of surgery for early breast cancer on local recurrence and 15-year survival: an overview of the randomised trials. Lancet. 2005;366(9503):2087-106. doi: 10.1016/S0140-6736(05)67887-7. PubMed PMID: 16360786.

2. van Dongen JA, Voogd AC, Fentiman IS, Legrand C, Sylvester RJ, Tong D, van der Schueren E, Helle PA, van Zijl K, Bartelink H. Long-term results of a randomized trial comparing breast-conserving therapy with mastectomy: European Organization for 
Research and Treatment of Cancer 10801 trial. J Natl Cancer Inst. 2000;92(14):1143-50. PubMed PMID: 10904087.

3. Fisher B, Anderson S, Bryant J, Margolese RG, Deutsch M, Fisher ER, Jeong JH, Wolmark N. Twenty-year follow-up of a randomized trial comparing total mastectomy, lumpectomy, and lumpectomy plus irradiation for the treatment of invasive breast cancer. N Engl J Med. 2002;347(16):1233-41. doi: 10.1056/NEJMoa022152. PubMed PMID: 12393820.

4. Deneve JL, Hoefer RA, Jr., Harris EE, Laronga C. Accelerated partial breast irradiation: a review and description of an early North American surgical experience with the intrabeam delivery system. Cancer Control. 2012;19(4):295-308. PubMed PMID: 23037497.

5. Vaidya JS, Joseph DJ, Tobias JS, Bulsara M, Wenz F, Saunders C, Alvarado M, Flyger HL, Massarut S, Eiermann W, Keshtgar M, Dewar J, Kraus-Tiefenbacher U, Sutterlin M, Esserman L, Holtveg HM, Roncadin M, Pigorsch S, Metaxas M, Falzon M, Matthews A, Corica T, Williams NR, Baum M. Targeted intraoperative radiotherapy versus whole breast radiotherapy for breast cancer (TARGIT-A trial): an international, prospective, randomised, non-inferiority phase 3 trial. Lancet. 2010;376(9735):91-102. doi: 10.1016/S0140-6736(10)60837-9. PubMed PMID: 20570343.

6. Veronesi U, Orecchia R, Maisonneuve P, Viale G, Rotmensz N, Sangalli C, Luini A, Veronesi P, Galimberti V, Zurrida S, Leonardi MC, Lazzari R, Cattani F, Gentilini O, Intra M, Caldarella P, Ballardini B. Intraoperative radiotherapy versus external radiotherapy for early breast cancer (ELIOT): a randomised controlled equivalence trial. 
Lancet Oncol. 2013;14(13):1269-77. doi: 10.1016/S1470-2045(13)70497-2. PubMed

PMID: 24225155.

Figure 1. Intraoperative photo of breast intraoperative radiation therapy utilizing the Intrabeam system and the Lone Star Retractor system used for self-retaining retraction of the skin away from the applicator.

\begin{tabular}{|l|c|c|c|}
\hline \multicolumn{4}{|c|}{ Table 1. Demographic and clinical features associated with aborted IORT. } \\
\hline \multicolumn{1}{|c|}{ Variable } & $\begin{array}{c}\text { Completed IORT } \\
(\mathbf{n = 1 2 4})\end{array}$ & $\begin{array}{c}\text { Aborted } \\
\text { IORT } \\
(\mathbf{n = 2 1})\end{array}$ & p-Value \\
\hline Age (years), mean \pm SD & $71.0 \pm 7$ & $69 \pm 7$ & 0.35 \\
\hline Tumor Size (cm), mean & $1.0(0.3-3.5)$ & $1.3(0.2-3.5)$ & 0.40 \\
\hline Additional Intraoperative Margin n (\%) & $59(48)$ & $17(81)$ & $\mathbf{0 . 0 0 5}$ \\
\hline Final Margin Positive n (\%) & $7(6)$ & $0(0)$ & 0.17 \\
\hline Sentinel Lymph Node Positive n (\%) & $8(6)$ & $2(9)$ & 0.77 \\
\hline Received Adjuvant WBRT n (\%) & $13(10)$ & $17(81)$ & $<\mathbf{0 . 0 0 1}$ \\
\hline $\begin{array}{l}\text { IORT intraoperative radiation therapy; SD standard deviation; WBRT whole breast radiation } \\
\text { therapy }\end{array}$ \\
\hline
\end{tabular}

\begin{tabular}{|l|c|}
\hline Table 2. Reasons for failure to complete planned IORT \\
\hline & $\mathbf{N = 2 1}$ \\
\hline Inadequate Skin-to-applicator distance & $15(71)$ \\
\hline Altered wire localization findings & $4(19)$ \\
\hline Equipment failure & $1(5)$ \\
\hline Hemodynamic instability & $1(5)$ \\
\hline IORT (intraoperative radiation therapy) & \\
\hline
\end{tabular}

Table 3. Completed versus Aborted IORT Cases by Surgeon.

\begin{tabular}{|c|c|c|c|}
\hline & $\begin{array}{c}\text { Completed } \\
(\mathbf{n}=124)\end{array}$ & $\begin{array}{c}\text { Failed to } \\
\text { Complete }\end{array}$ & Failed to Complete \\
& due to \\
\hline
\end{tabular}




\begin{tabular}{|c|c|c|c|}
\hline & & $\begin{array}{l}(\mathrm{n}=21) \\
\mathrm{n}(\%)\end{array}$ & $\begin{array}{c}\text { Inadequate Skin- } \\
\text { to-applicator } \\
\text { distance } \\
\text { n (\% of failed to } \\
\text { complete })\end{array}$ \\
\hline Surgeon A & 67 & $2(3)$ & $1(50)$ \\
\hline Surgeon B & 26 & $1(4)$ & $1(100)$ \\
\hline Surgeon C & 21 & $8(27)$ & $7(88)$ \\
\hline Surgeon D & 7 & $7(50)$ & $4(58)$ \\
\hline Surgeon E & 3 & $3(50)$ & $2(67)$ \\
\hline
\end{tabular}




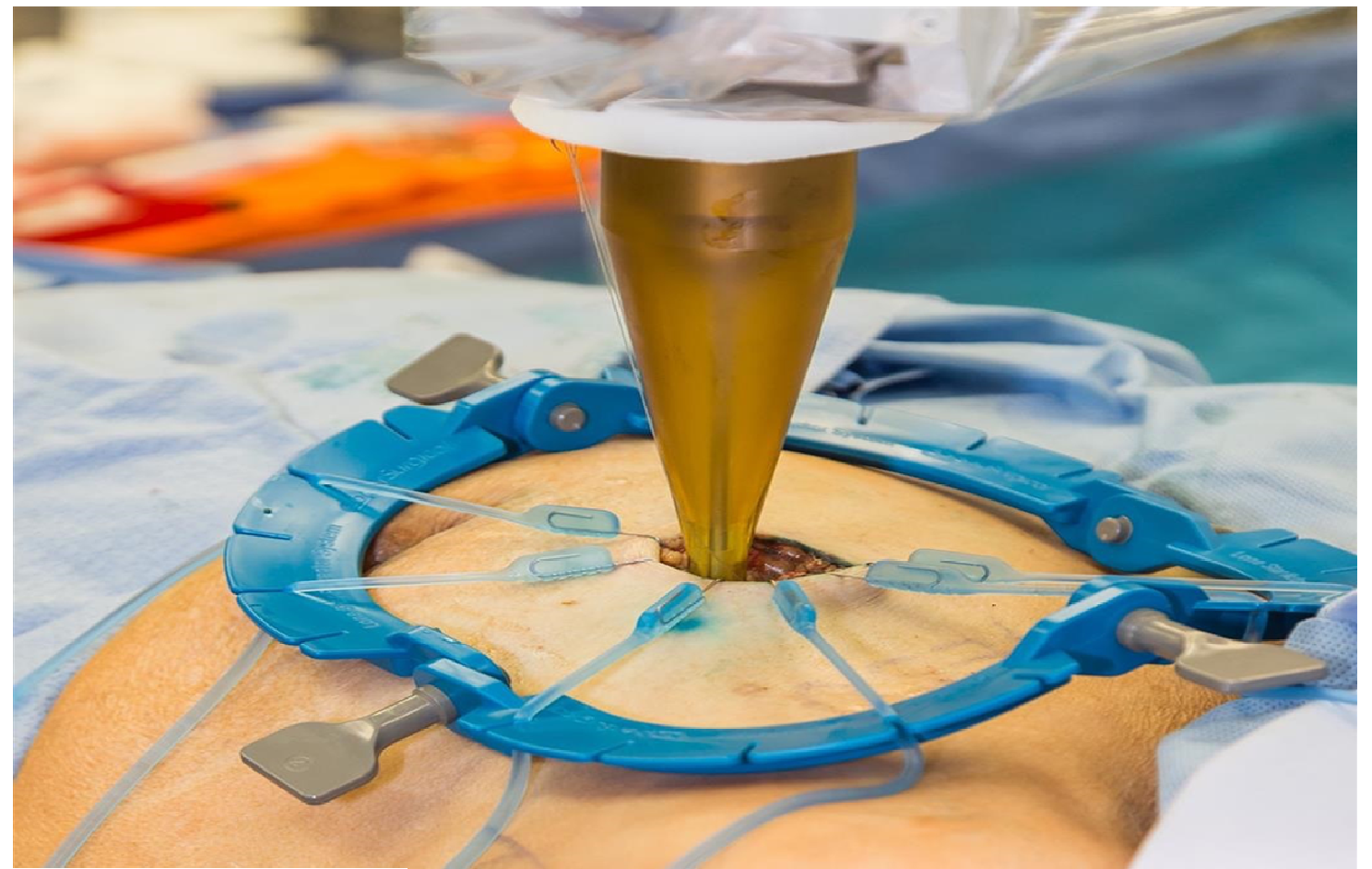

JSO Failed IORT Figure 1 . 